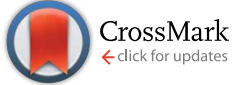

Cite this: RSC Adv., 2016, 6, 30755

Received 3rd February 2016

Accepted 7th March 2016

DOI: $10.1039 / \mathrm{c} 6 \mathrm{ra03239g}$

www.rsc.org/advances

\section{Influence of clay-nanofiller geometry on the structure and properties of poly(lactic acid)/ thermoplastic polyurethane nanocomposites}

\author{
I. Kelnar, ${ }^{\text {aa }}$ J. Kratochvíl, ${ }^{a}$ I. Fortelný, ${ }^{a}$ L. Kaprálková, ${ }^{a}$ A. Zhigunov, ${ }^{a}$ M. Nevoralová, ${ }^{a}$ \\ M. Kotrisováb and V. Khunováb
}

The effect of two clays with different geometries, viz. organophilized montmorillonite nanoplatelets (OMMT) and halloysite nanotubes (HNT), on the structure and properties of poly(lactic acid) (PLA)/thermoplastic polyurethane (TPU) 80/20 and 70/30 has been studied. The reinforcement of both the components and the blend is higher for OMMT due to its higher specific surface area and aspect ratio. The effect of both nanofillers (NFs) on the structure, reflected in a decrease of the dispersed TPU size, is comparable. A larger effect on the viscosity by OMMT is eliminated by its more marked less favourable localization in the dispersed phase in comparison with HNT. The fact that the toughness of the HNT-containing nanocomposite (NC) is markedly higher (with maximum at $1 \%$ HNT) than that with oMMT is in contradiction with PLA NC. The results indicate that the application of nanofillers can lead to a polymer system with a balanced mechanical behaviour. However, a different impact of NFs on the polymer components and interface parameters may also lead to antagonistic effects. The influence of NFs, including their geometry, on the polymer blend is more complex in comparison with single-matrix nanocomposites.

\section{Introduction}

PLA is a biodegradable and biocompatible polymer with high stiffness and tensile stress. However, its range of applications in replacing traditional commodity/engineering plastics and in the bio-medicinal field are limited by low toughness. ${ }^{1}$ Extensive work has been published in the area of impact modification using different elastomers, e.g. TPU. ${ }^{2-6}$ Due to low compatibility, various compatibilization methods have to be applied. ${ }^{5-7}$ An effective approach is, in particular, toughening using various copolymers based on biodegradable polyesters which show a high effect at a low content, which leads to a less significant decrease of stiffness and strength. ${ }^{8}$ Recently, it has been shown that the addition of various nanofillers can significantly enhance the mechanical parameters of toughened systems. ${ }^{\mathbf{8 - 1 2}}$ The combination of impact modification and NC concepts do not only combine a high reinforcing effect with increased energy dissipation, but synergistic effects can also occur. This may result in a situation when the addition of NF, usually not affecting the toughness of $\mathrm{NC}^{\mathbf{1 3}}$ may lead to increased toughness in an impact-modified multicomponent system. The reason is a combination of the compatibilizing effect, leading to

anstitute of Macromolecular Chemistry, Academy of Sciences of the Czech Republic, Heyrovského nám. 2, 16206 Praha, Czech Republic. E-mail: kelnar@imc.cas.cz

${ }^{b}$ The Slovak University of Technology, Faculty of Chemical and Food Technology, Radlinského 9, 81237 Bratislava, Slovakia the refinement of a dispersed impact modifier, with the formation of an effective morphology of the dispersed phases, e.g. those with NF localized at the interface ${ }^{\mathbf{1 2 , 1 4}}$ or with interconnected inclusions. ${ }^{15}$ The replacement of a low-modulus polymeric compatibilizer with reinforcing rigid particles is also beneficial. Until now, oMMT has mostly been applied..$^{9-12}$ In the case of modified PLA, including toughening by various TPUs ${ }^{16}$ good effectivity was usually found with a particular NF. ${ }^{15,17,18}$ However, the disadvantage of such an NF is a relatively low reinforcing ability.

Another natural NF with a fair reinforcing ability is $\mathrm{HNT}^{\mathbf{1 9 , 2 0}}$ which also has a pronounced effect on biodegradable polymers, including PLA, ${ }^{21,22}$ even without any modification. However, the application of HNT in multicomponent systems is still in its infancy. ${ }^{23-28}$

This work deals with the effect of oMMT and HNT on the structure and properties of a PLA/commercial TPU system. The goal was a more detailed study of the effect of nanofiller content and a comparison of the reinforcing/structure-directing abilities of nanosilicates with various geometries.

\section{Experimental}

\subsection{Materials}

Poly(lactic acid) (PLA) Ingeo 2002D with a D-isomer content of $4.3 \%, M_{\mathrm{w}}$ of $2.53 \times 10^{5} \mathrm{~g} \mathrm{~mol}^{-1}$, melt flow rate of $6 \mathrm{~g} / 10 \mathrm{~min}$ (190 ${ }^{\circ} \mathrm{C} / 2.16 \mathrm{~kg}$ ), and density of $1.24 \mathrm{~g} \mathrm{~cm}^{-3}$ was obtained from 
Nature Works, Minnetonka, USA. Thermoplastic polyurethane (TPU) Desmopan ${ }^{\circledR} 460$ was obtained from Bayer. Halloysite nanotubes (HNT) were obtained from Sigma Aldrich (USA). Clay based on natural montmorillonite: Cloisite 30B modified with alkyl bis(2-hydroxyethyl)dimethylammonium chloride $90 \mathrm{meq} /$ $100 \mathrm{~g}$, with alkyl derived from tallow (oMMT) was obtained from Southern Clay Products, Inc.

\subsection{Sample preparation}

Prior to mixing, TPU, PLA, and HNT were dried at $45{ }^{\circ} \mathrm{C}, 85^{\circ} \mathrm{C}$, and $70{ }^{\circ} \mathrm{C}$, respectively, in a vacuum oven for $12 \mathrm{~h}$. Mixing was carried out in a co-rotating segmented twin-screw extruder (L/D 40) Brabender TSE 20 (Brabender GmbH, Duisburg, Germany) at $400 \mathrm{rpm}$, and the temperatures of the respective zones (from feeding to die) were $185,190,190,190,190$, and $195{ }^{\circ} \mathrm{C}$. Dogbone specimens (gauge length $40 \mathrm{~mm}$ ) were formed through injection using a laboratory micro-injection moulding machine (DSM). The barrel and mould temperatures were $200{ }^{\circ} \mathrm{C}$ and 30 ${ }^{\circ} \mathrm{C}$, respectively.

\subsection{Testing}

Tensile tests were carried out using an Instron 5800 (Instron, UK) apparatus at $22{ }^{\circ} \mathrm{C}$ and crosshead speed of $20 \mathrm{~mm} \mathrm{~min}^{-1}$. At least eight specimens were tested for each sample. The Young's modulus $(E)$, maximum stress $\left(\sigma_{\mathrm{m}}\right)$, and elongation at break $\left(\varepsilon_{\mathrm{b}}\right)$ were evaluated. The corresponding variation coefficients did not exceed $10 \%, 2 \%$ and $20 \%$, respectively.

Tensile impact strength, $a_{\mathrm{t}}$, was measured on specimens notched on one-side using a CEAST Resil impact junior hammer (CEAST S.p.A., Torino, Italy) with an energy of $4 \mathrm{~J}$ (variation coefficient 10-15\%). The reported values are averages of twelve individual measurements. Dynamic mechanical analysis (DMA) was performed in single-cantilever mode using a DMA DX04T (RMI, Pardubice, Czech Republic) apparatus at 1 $\mathrm{Hz}$ and a heating rate of $1{ }^{\circ} \mathrm{C} \mathrm{min}^{-1}$ from -120 to $250{ }^{\circ} \mathrm{C}$.

DSC analysis was carried out using Perkin-Elmer 8500 DSC apparatus. Cyclohexane and indium were used for calibration. The instrument was cooled with liquid nitrogen using an $\mathbf{L N}_{2}$ accessory at a set-point of $-30{ }^{\circ} \mathrm{C}$ and flushed with dry nitrogen as a purge gas. The samples were scanned from 0 to $200{ }^{\circ} \mathrm{C}$ at 10 ${ }^{\circ} \mathrm{C} \min ^{-1}$. The melting point and cold crystallization temperature were identified as a maximum and minimum of the melting endotherm and crystallization exotherm, respectively. A value of $93.1 \mathrm{~J} \mathrm{~g}^{-1}$ was used as the heat of melting of $100 \%$ crystalline PLA $^{29}$ in calculating its crystallinity.

\subsection{Characterization of structure}

The structure was examined using scanning electron microscopy (SEM) with a Quanta 200 FEG (FEI, Czech Republic) microscope. The injection-moulded specimens were broken under liquid nitrogen and etched in $20 \% \mathrm{NaOH}$ for $20 \mathrm{~min}$. The size of the dispersed PLA particles was investigated using a MINI MOP image analyzer (Kontron Co., Germany). At least 200 particles were evaluated in each sample. For the transmission electron microscope Tecnai G2 Spirit (FEI, Czech Republic) observations, ultrathin $(60 \mathrm{~nm})$ sections were prepared under liquid nitrogen using an Ultracut UCT ultramicrotome (Leica Microsystems GmbH, Austria).

Wide-angle X-ray scattering (WAXS) experiments were performed using a pinhole camera (Molecular Metrology System, Rigaku, Japan) attached to a microfocused X-ray beam generator (Osmic MicroMax 002) operating at $45 \mathrm{kV}$ and $0.66 \mathrm{~mA}$ (30 $\mathrm{W})$. The camera was equipped with an interchangeable imaging plate of $23 \times 25 \mathrm{~cm}$ (Fujifilm). The experimental setup covered a momentum transfer $(q)$ range of $0.25-3.5 \AA^{-1} \cdot q=(4 \pi / \lambda) \sin \theta$, where $\lambda=1.54 \AA$ is the wavelength and $2 \theta$ is the scattering angle. Calibrations of the centre and sample-to-detector distance were made using Si powder. Samples were measured in the transmission mode. Due to their isotropic character, 2D diffractograms were transformed into $1 \mathrm{D}$ using the whole 0$360^{\circ}$ azimuthal range.

\subsection{Rheological characterization}

Rheological characterization was conducted using an ARES apparatus (Rheometric Scientific, Piscataway, NJ) with a parallel-plate geometry at $190{ }^{\circ} \mathrm{C}$ using an oscillatory shear deformation at a frequency range of 0.1-100 $\mathrm{rad} \mathrm{s}^{-1}$. The amplitude of the oscillation was $0.1 \%$, i.e. within the range of the linear viscoelasticity of all of the studied materials.

\section{Results and discussion}

\subsection{Effect of nanofiller on structure}

The structure-directing ability of both nanofillers reflected in the effect on the size of the dispersed TPU droplets is shown in Fig. 1. From Fig. 2, it is obvious that the addition of both NFs to the PLA/TPU 80/20 system leads to a comparable decrease of particle size. The most significant difference is the higher polydispersity of the HNT-modified system (Fig. 1c). With a 30\% content of TPU, the initial particle size in the HNT-free blend is higher $(\sim 2.8 \mu \mathrm{m})$, which corresponds to a higher TPU content. However, the relative decrease (to $\sim 1 \mu \mathrm{m}$ ) is similar to the $80 / 20$ system (not shown). The dependence of the particle size on the nanofiller content in Fig. 2 is similar to the emulsification curves found for oMMT. ${ }^{\mathbf{1 4}}$ Considering the higher specific surface area of MMT in comparison with HNT $\left(800 \mathrm{~m}^{2} \mathrm{~g}^{-1} v s\right.$. $\sim 80 \mathrm{~m}^{2} \mathrm{~g}^{-1}$ ), a similar, or even slightly lower "compatibilizing" effect of the layered silicate seems to be surprising. Moreover, in this respect, many other factors, such as the degree of dispersion, localization, change in component viscosities and their ratios caused by the nanofiller, etc., should be taken into account.

Fig. 3 shows beneficial ${ }^{14}$ interfacial localization in both cases, but it is more significant for oMMT. This undoubtedly supports the "compatibilizing" effect of NF. This figure also shows more significant localization of oMMT inside the dispersed phase in comparison with HNT. This difference is quite important with respect to possible effects on the dynamic phase behaviour. A high content of well dispersed oMMT inside TPU apparently increases its viscosity (see below), which hinders particle break-up in the course of mixing. This obviously eliminates the above mentioned effect of more significant 

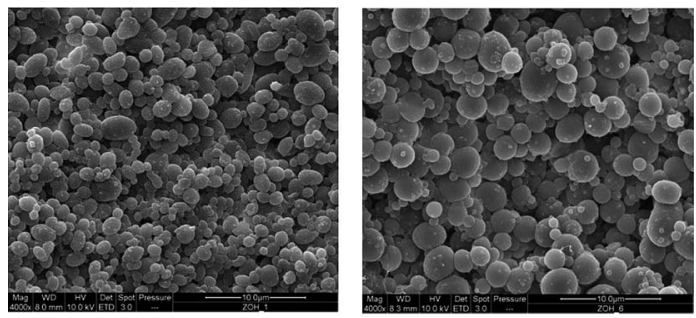

a)

d)
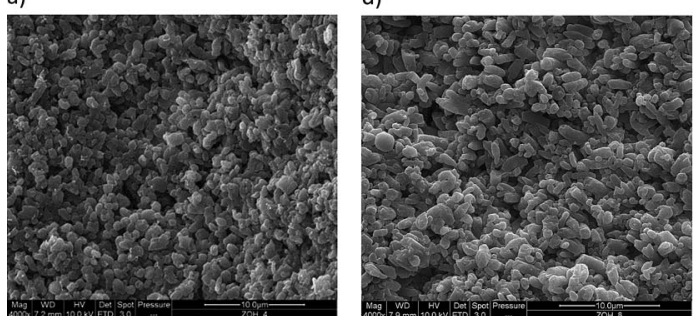

e)

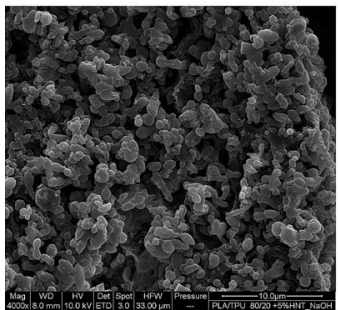

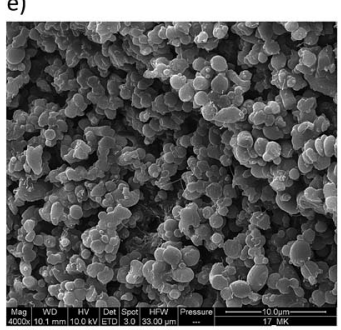

Fig. 1 SEM images of (a) PLA/TPU 80/20 blend, (b) with 5 phr OMMT, and (c) with 5 phr HNT, and (d) PLA/TPU 70/30 blend, (e) with 5 phr OMMT, and (f) 5 phr HNT.

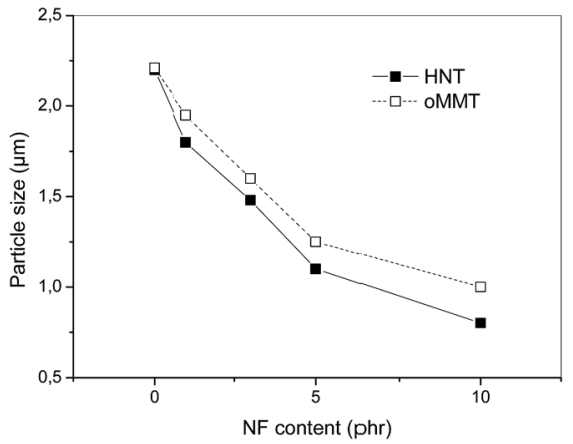

Fig. 2 Effect of nanofiller content on size of dispersed TPU in the nanocomposite with PLA/TPU 80/20 matrix.

interfacial localization of oMMT and seems to be the reason for the relatively lower effectivity of oMMT in comparison with HNT. Probably, this effect is only partially compensated by the higher viscosity of the oMMT containing system. At the same time, the lower specific surface area of HNT is apparently compensated by a relatively low increase of viscosity, which is beneficial in the case of HNT localized in the dispersed phase. Moreover, the mentioned more significant content of HNT in the matrix may lead to a favourable increase of matrix viscosity and decrease of viscosity ratio, which supports the break-up of the dispersed phase. Taking into account the more marked

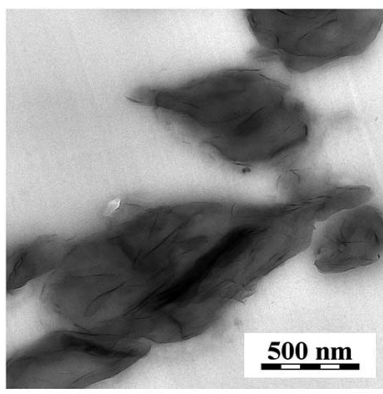

a)

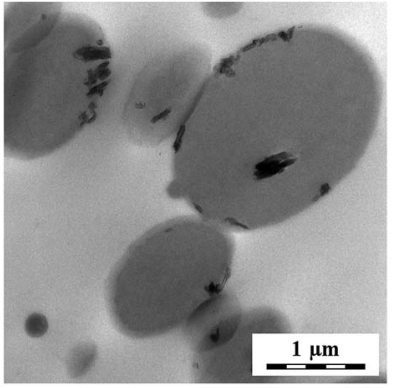

b)

Fig. 3 TEM images of (a) PLA/TPU/OMMT 70/30/3, and (b) PLA/TPU/ HNT 80/20/3 systems.

effect of HNT on PLA degradation (see below), this effect can be considered to take place in the early stage of mixing.

\subsection{Dynamic mechanical analysis}

The DMA results (Fig. 4) indicate that both oMMT and HNT decrease the $T_{\mathrm{g}}$ of PLA and TPU; this effect is more significant for oMMT. Such a decrease has also been found for single NC (not shown). It obviously prevails over the possible effect on $T_{\mathrm{g}}$ of the enhanced compatibility of the polymer components caused by NFs. The fact that the decrease of the $T_{\mathrm{g}}$ of TPU is more pronounced in comparison with PLA $\left(\sim 5{ }^{\circ} \mathrm{C} v s . \sim 1{ }^{\circ} \mathrm{C}\right)$ apparently corresponds to the more significant presence of both NFs in the TPU phase (Fig. 3). Although the effect of NFs on glass transition is still not fully understood and may depend e.g. on changed interparticle distances, ${ }^{30}$ a more significant decrease of the $T_{\mathrm{g}}$ of both components in the case of oMMT may be caused by a plasticizing effect of the $\mathrm{OH}$ groups of the clay modifier. ${ }^{31}$ The more distinct shoulder between $T_{\mathrm{g}}$ peaks with an increasing NF content, observed especially for oMMT, corresponds to the overall immobilization of polymer chains by NFs and is in accordance with the higher reinforcing effect of oMMT.

\subsection{Mechanical properties}

The results of mechanical testing have shown a higher $E$ of both of the PLA- and PLA/TPU-matrix nanocomposites containing oMMT in comparison with the analogous HNT-systems (Fig. 5). This higher reinforcing effect of oMMT is undoubtedly a consequence of the higher specific surface area and aspect ratio of oMMT ( $>100$ for oMMT, $<25$ for HNT). The relative increase of $E$ with NF content (Fig. 5) is comparable for all of the 

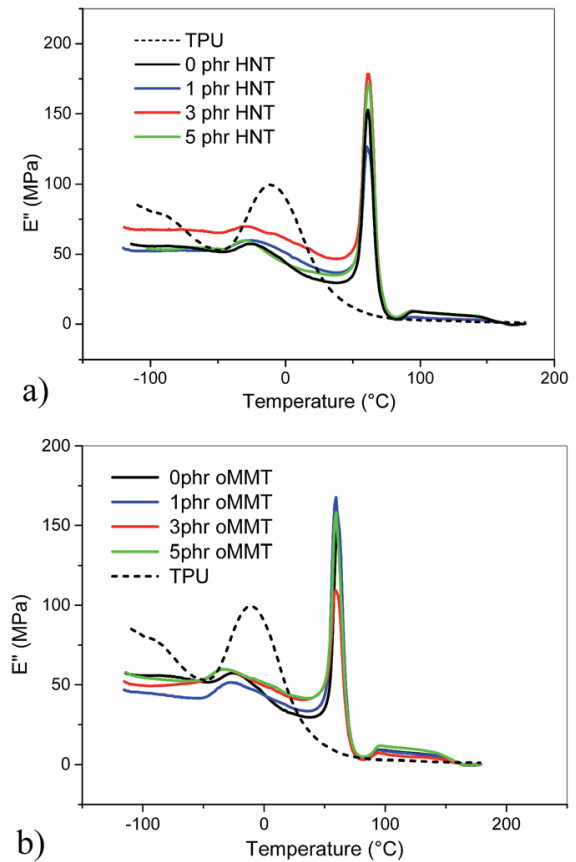

Fig. 4 Temperature dependence of the loss modulus of PLA/TPU 70/ 30 blend containing (a) HNT, and (b) OMMT.

systems studied, i.e. PLA, PLA/TPU 80/20 and 70/30. This is somewhat unexpected because NF localization inside the rubbery component at the expense of the matrix is less favourable; a higher rigidity of dispersed TPU containing NF has lower effect on $E$ in comparison with the direct reinforcement of the matrix as indicated by model calculations using the Kerner model. ${ }^{32}$ We also consider the influence of the contribution of the interfacial localization of oMMT on the interphase - the formation of a rigid regular shell around TPU (Fig. 3), and also possibly affecting the crystallinity in this area. As a result, the effect of NFs on the stiffness of the multiphase system is more complex than the mere reinforcement of the components. In the case of HNT, lower $E$ values, in comparison with the analogous oMMT-containing system, occur in spite of a more marked presence of HNT in PLA and a slightly higher PLA crystallinity (see below). This corresponds to the lower reinforcing ability of HNT in comparison with oMMT, which is obvious from the $E$ values of PLA-matrix NC (Fig. 5).

Fig. 6 shows a rather different effect of both of the NFs' content on tensile strength, especially in the dependence on the matrix composition. Although higher values for oMMT have been found in all systems, the effect of the NF content is different. In neat PLA, a slight increase with oMMT content and decrease with HNT content has been found. This is in accordance with the fact that NF-reinforcement predominantly consists of an increase of stiffness. ${ }^{33}$ In the case of the PLA/TPU 80/20 matrix, Fig. 6 shows a practically unaffected tensile strength in the whole range of NF content (up to 10\%) for HNT and a slight maximum at 5\% content for oMMT. This may correspond to reduced PLA crystallinity $\left(\mathrm{CR}_{\mathrm{p}}\right.$ in Table 1$)$ at a high oMMT content in comparison with the analogous HNTcontaining system. Compared with neat PLA, the increase of the strength of the blends with both NFs is more marked. We consider the positive effect of the finer structure and reinforcement of TPU (see the predominant localization of oMMT in dispersed TPU, Fig. 3) because the addition of a low-modulus elastomer decreases strength significantly. Surprisingly, in the 70/30 system only a slight decrease, more significant for HNT, was found. Although the reinforcing effect seems to be partially eliminated by the higher particle size in the 70/30 system (Fig. 2), this difference undoubtedly confirms the complex effect of NFs in a two-component matrix nanocomposite.

On the contrary, higher toughness values were found in the HNT-containing systems (Fig. 7). In the PLA nanocomposite, the increase corresponding to the results of other authors ${ }^{13}$ indicates the fair dispersion of NF. The slightly lower toughness in the case of oMMT may correspond to a higher reinforcement and thus a higher overall degree of unfavourable immobilization of the polymer chains. A relatively low gain in toughness due to $20 \%$ and especially $30 \%$ TPU addition indicates the limited toughening ability of the TPU applied. In this respect, a marked increase of PLA/TPU 80/20 toughness with 1\% HNT is important. This undoubtedly indicates certain synergistic effects of the nanofiller on the multi-component matrix NC found also by others ${ }^{8}$, mostly with platelets and particles, especially in the case of their more significant interfacial localization. In PLA/TPU 80/20, this can be explained by the

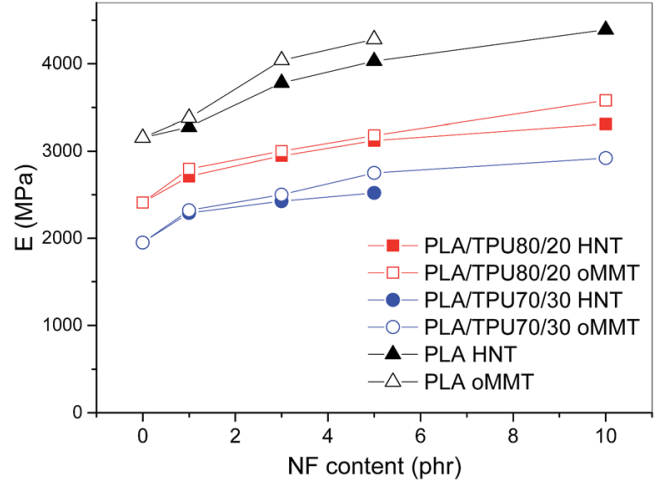

Fig. 5 Effect of nanofiller content on modulus.

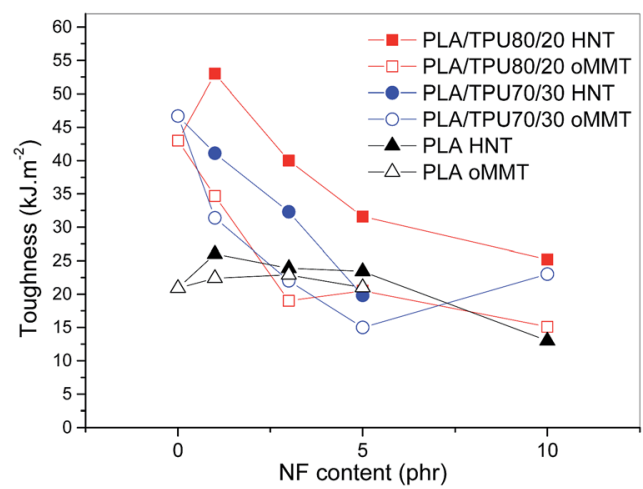

Fig. 6 Effect of nanofiller content on tensile strength. 
Table 1 DSC analysis of PLA/TPU composites; values = averages of four measurements

\begin{tabular}{|c|c|c|c|c|c|c|c|}
\hline \multirow[b]{2}{*}{ Composition } & \multirow[b]{2}{*}{ Filler (phr) } & \multirow[b]{2}{*}{$T_{\mathrm{g}},{ }^{\circ} \mathrm{C}$} & \multicolumn{2}{|l|}{ Exo } & \multicolumn{2}{|l|}{ Endo } & \multirow[b]{2}{*}{$\mathrm{CR}_{\mathrm{p}}, \%$} \\
\hline & & & $T_{\mathrm{c}},{ }^{\circ} \mathrm{C}$ & $\mathrm{CR}_{\mathrm{c}}, \%$ & $T_{\mathrm{m}},{ }^{\circ} \mathrm{C}$ & $\mathrm{CR}_{\mathrm{m}}, \%$ & \\
\hline PLA/TPU 80/20 & 0 & 60.0 & 106.6 & 24.6 & 149.0 & 29.3 & 4.7 \\
\hline \multirow[t]{4}{*}{ PLA/TPU 80/20 + HNT } & 1 & 59.1 & 106.2 & 24.2 & 150.3 & 29.3 & 5.2 \\
\hline & 3 & 59.9 & 105.9 & 26.4 & 149.2 & 31.0 & 4.6 \\
\hline & 5 & 60.4 & 105.6 & 25.9 & 149.8 & 30.9 & 5.0 \\
\hline & 10 & 59.2 & 107.7 & 29.1 & 148.6 & 33.3 & 4.2 \\
\hline \multirow[t]{4}{*}{ PLA/TPU 80/20 + oMMT } & 1 & 59.7 & 105.1 & 22.8 & 148.0 & 28.2 & 5.4 \\
\hline & 3 & 58.0 & 105.7 & 25.4 & 149.6 & 29.6 & 4.2 \\
\hline & 5 & 58.1 & 109.7 & 28.3 & 149.9 & 30.9 & 2.6 \\
\hline & 10 & 57.4 & 109.8 & 30.8 & 152.1 & 31.5 & 0.7 \\
\hline
\end{tabular}

lower content of HNT inside the TPU inclusions and its presence at the interface (Fig. 3).

From Fig. 7, it follows that the toughness of all other PLA/ TPU/nanofiller systems (80/20 with oMMT and 70/30 with both NF) decreases monotonously, less markedly with HNT. The main reason is NF localization inside TPU which increases its rigidity and reduces the toughening effect. This negative effect is more marked in the oMMT system with its increased content inside TPU and higher reinforcing ability. As a result, a more significant decrease of toughness with oMMT content has been found. This is in accordance with other authors who found a similar decrease even in the case of the absence of clay in a dispersed phase. ${ }^{34,35}$ These results indicate that the influence of clay on multicomponent systems may lead to rather antagonistic effects and further confirms the complex effect NF has on these systems. Certain negative effects in the oMMTsystem can also originate from reduced PLA crystallinity, which may lead to less favourable energy-absorbing microdeformations. ${ }^{36}$ Further differences, especially in the crystalline structure, between the oMMT- and HNT-systems are obvious from WAXS. Different degrees of the immobilization of polymer chains is also shown by DMA (see above). Finally, strain at break (Fig. 8) decreases predominantly with a higher NF content in accordance with increased rigidity, but it partially follows the synergistic increase of toughness with $1 \% \mathrm{HNT}$, surprisingly also in the case of oMMT. All the mentioned facts, especially the

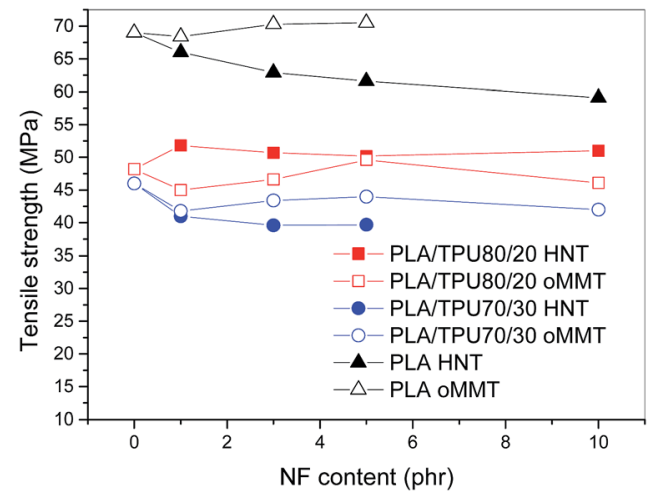

Fig. 7 Effect of nanofiller content on toughness. differences between the HNT- and oMMT-systems, also confirm the complex and different effects on multiphase NC of both NFs with dissimilar geometry.

\subsection{Differential scanning calorimetry}

A typical DSC thermogram of the studied system is shown in Fig. 9. The glass transition temperature $T_{\mathrm{g}}$ of PLA at about $60^{\circ} \mathrm{C}$ is accompanied by a distinct peak of enthalpic relaxation. Immediately after the system crosses $T_{\mathrm{g}}$, the DSC trace shows a characteristic feature of PLA in multicomponent systems, i.e. cold crystallization on heating demonstrated by the deep exotherm with a minimum at about $106{ }^{\circ} \mathrm{C}$. The melting endotherm at about $150{ }^{\circ} \mathrm{C}$ reflects total PLA crystallinity, i.e. the sum of crystallinity of the as-prepared sample and that coming from the cold crystallization.

The results of the DSC analysis are summarized in Table 1 as averages of four measurements of the glass transition temperature $T_{\mathrm{g}}$, the temperature of minimum of the cold crystallization exotherm $T_{\mathrm{c}}$, i.e. of maximum crystallization rate, the crystallinity connected with cold crystallization $\mathrm{CR}_{\mathrm{c}}$, the melting temperature $T_{\mathrm{m}}$, the total crystallinity $\mathrm{CR}_{\mathrm{m}}$, and the crystallinity of the as-prepared sample $\mathrm{CR}_{\mathrm{p}}$.

Fig. 9 shows that the cold-crystallization exotherm passes smoothly to the melting endotherm as the processes of cold crystallization and melting partially overlap. It is thus impossible to unambiguously determine the areas of these two peaks.

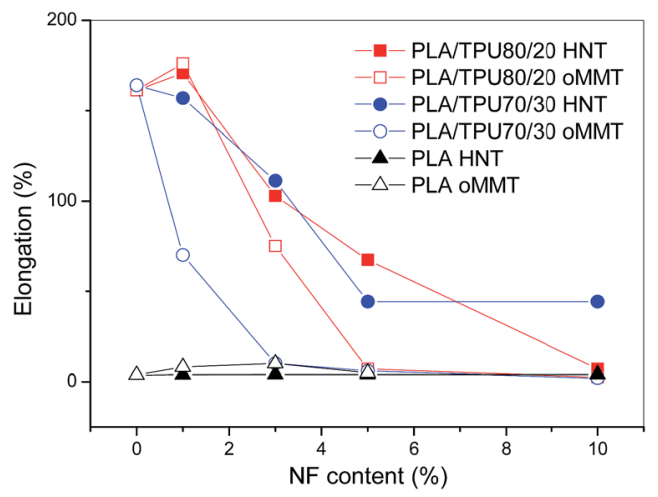

Fig. 8 Effect of nanofiller content on elongation. 


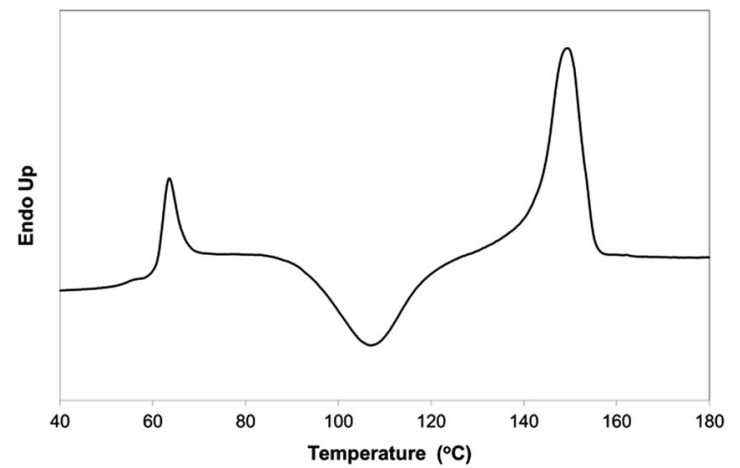

Fig. 9 DSC thermogram of the system PLA/TPU 80/20 + 3 phr HNT.

Therefore, an integration was performed by leading the baseline between 70 and $160{ }^{\circ} \mathrm{C}$. The $\mathrm{CR}_{\mathrm{c}}$ value in Table 1 was obtained from the area of the exotherm between $70{ }^{\circ} \mathrm{C}$ and the crosssection of the baseline with the DSC trace. Analogously, the $\mathrm{CR}_{\mathrm{m}}$ value was obtained from the area of the endotherm between the cross-section and $160{ }^{\circ} \mathrm{C}$. The as-prepared crystallinity $\mathrm{CR}_{\mathrm{p}}$, as the difference $\mathrm{CR}_{\mathrm{m}}-\mathrm{CR}_{\mathrm{c}}$, results in the neat thermal balance of the integration between 70 and $160{ }^{\circ} \mathrm{C}$.

The results in Table 1 indicate that, with the exception of slightly rising $\mathrm{CR}_{\mathrm{c}}$ and $\mathrm{CR}_{\mathrm{m}}$, the determined parameters are virtually independent of the added HNT concentration. On the other hand, some dependence on the concentration of the added oMMT has been found. The $T_{\mathrm{g}}$ smoothly decreases with increasing oMMT content. The values of $T_{\mathrm{c}}, \mathrm{CR}_{\mathrm{c}}, T_{\mathrm{m}}$, and $\mathrm{CR}_{\mathrm{m}}$ have a generally increasing trend, however, with a minimum at about 1\% oMMT. The crystallinity of the as-prepared samples, $\mathrm{CR}_{\mathrm{p}}$, passes through a maximum at about $1 \%$ and then decreases sharply with the oMMT content. Anyway, all of the asprepared samples show very low PLA crystallinity of about 1$5 \%$. On heating, this crystallinity increases to about $30 \%$ due to the intensive cold crystallization.

\subsection{Wide-angle $X$-ray scattering analysis}

Fig. 10 shows 1D diffractograms of all samples. The peaks are rather broad, which corresponds to small crystallites. Sizes calculated by the Scherrer equation

$$
L=\frac{K \lambda}{\beta \cos \theta},
$$

are in the range from 10 to $15 \AA$. The crystallinities of PLA in the presence of 1\% HNT and oMMT are the same, in accordance with the $\mathrm{CR}_{\mathrm{p}}$ values in Table 1 . The peak positions also coincide. Therefore, the differences in the mechanical properties at low NF content originate predominantly from the different reinforcing effects of both nanofillers.

It is hard to speculate about the decomposed peak's position when it originates from such broad spectra. Nevertheless, analysis shows that, in the case of the nanofiller-free PLA/TPU $80 / 20$ sample, the position of the first peak is shifted to lower angles. We can exclude possible TPU crystallization, as this should produce a shift to higher angles..$^{37,38}$ A possible

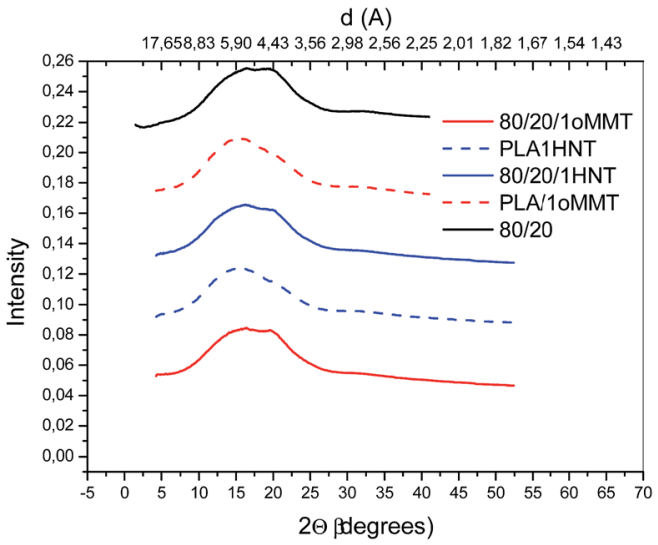

Fig. 10 Wide-angle $X$-ray scattering patterns of PLA and PLA/TPU nanocomposites.

explanation could be in partly transformed PLA into the $\alpha$ crystalline form, the most common and stable polymorph of PLA. During such transformation, a diffraction peak at $2 \theta=$ 14.8 appears. ${ }^{39}$ This effect is more pronounced when the oMMT filler is added. It is also seen that HNT produces rather the opposite effect. Better chain organization ( $\alpha$-form) leads to improved mechanical properties. This difference, especially if possibly localized in the vicinity of the TPU inclusions, may be a further reason for the differences in mechanical behaviour between PLA/TPU containing both NFs (see above).
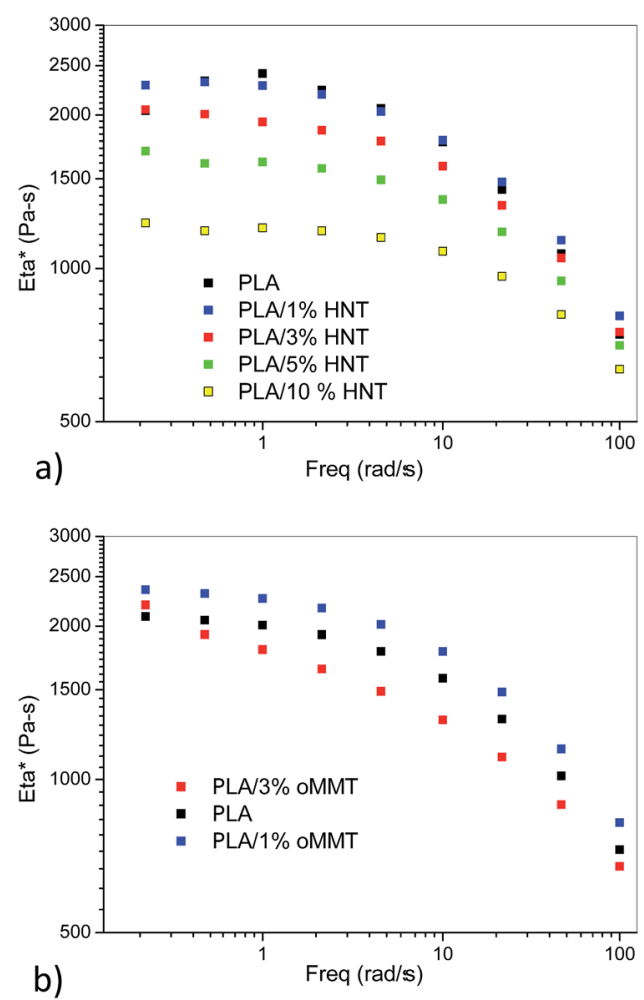

Fig. 11 Effect of NF addition on complex viscosity at $190^{\circ} \mathrm{C}$ of (a) PLA/ HNT and (b) PLA/OMMT. 

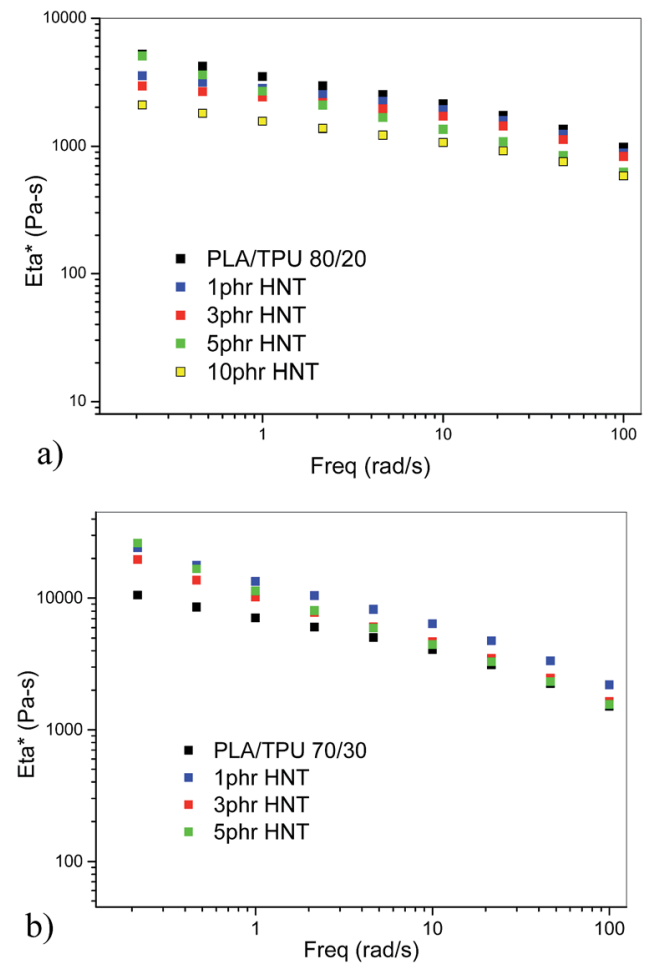

Fig. 12 Effect of NF addition on complex viscosity of the PLA/TPU/ HNT system at $190^{\circ}$ : (a) $80 / 20$ and (b) $70 / 30$.

\subsection{Effect of nanofillers on rheology of components and blends}

Fig. 11a shows that the viscosity of the PLA/HNT nanocomposites decreases with an increasing content of HNT in contrast to the common behaviour of polymer melts containing hard fillers. The most plausible explanation of this effect seems to be the degradation of PLA due to the presence of HNT. This was confirmed by size exclusion chromatography showing an approximately $10 \%$ decrease of molecular weight in the composite with $10 \%$ HNT.

The comparison of the dependence of $\eta^{*}$ on the frequency for neat PLA and the PLA/OMMT nanocomposite (Fig. 11b) indicates that, in the nanocomposite with $1 \%$ of oMMT an increase of $\eta^{*}$ due to the presence of hard fillers prevails over the decrease of $\eta^{*}$ caused by degradation. The degradation of PLA by the presence of a higher oMMT content also causes a lower viscosity of the nanocomposite at moderate and high frequencies.

At low frequencies, a higher $\eta^{*}$ of the nanocomposites containing 3\% OMMT apparently indicates the tendency of oMMT to form a weak physical network in the PLA matrix. Its contribution to $\eta^{*}$ competes with the effect of the degradation which is lower in comparison with the HNT-systems.

In the case of the PLA/TPU (80/20)/HNT system (Fig. 12a), rheological behaviour, especially the lower reduction of $\eta^{*}$ with HNT content, indicates a certain competition between the effect of the amount of HNT on the degradation of the component, on the one hand, and the tendency to increase $\eta^{*}$ by energy
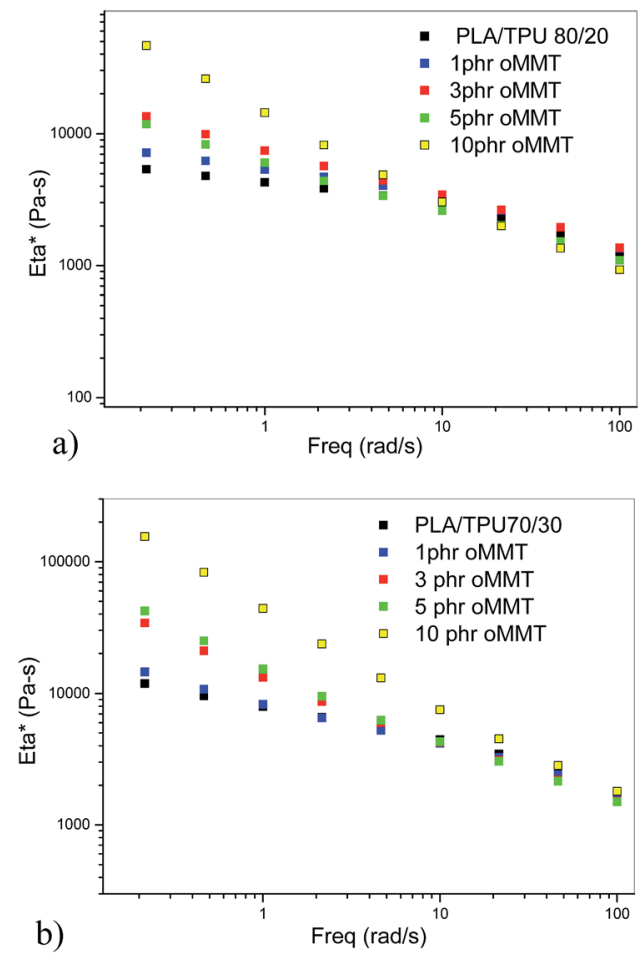

Fig. 13 Effect of NF addition on complex viscosity of the PLA/TPU/ OMMT system at $190^{\circ}$ : (a) $80 / 20$ and (b) 70/30.

dissipation in the presence of the fillers and formation of a physical network on the other. This is shown by the curves for PLA/TPU and the nanocomposites with 0.5 and $5 \%$ of HNT.

The dependence of $\eta^{*}$ on the frequency for PLA/TPU (70/30) filled with HNT (Fig. 12b) also demonstrates competition between the effect of the blend degradation and viscosity increase by the presence of hard fillers. The direct effect on the viscosity increase and the formation of a physical network is more pronounced in the PLA/TPU (70/30) blends in comparison with the 80/20 systems. Similarly to PLA/OMMT, the degradation of PLA/TPU/OMMT (Fig. 13a and b) is also less marked in comparison with the analogous HNT-system.

In the case of the oMMT-systems, Fig. 13a and b show an increase of viscosity with the concentration of the added clays in the region of low frequencies. The increase of viscosity with the amount of clay is more pronounced for the PLA/TPU (70/30) blend. The effect of the clay on the blend degradation is indicated by the values of $\eta^{*}$ in the region of high frequencies. Comparison of the effect of the addition of HNT and oMMT on the rheological properties of the PLA/TPU blends shows that the increase of $\eta^{*}$ at low frequencies is more pronounced in the PLA/TPU/OMMT than in the PLA/TPU/HNT nanocomposites. The decrease of $\eta^{*}$ with frequency is more pronounced for the PLA/TPU/OMMT than for the PLA/TPU/HNT nanocomposites.

\section{Conclusions}

The results indicate a comparable effect of the platy oMMT and tubular HNT clays on the size of dispersed TPU in the PLA/TPU 
matrix, i.e. a decrease of particle size with increasing NF content. The reinforcing effect is higher for oMMT in both the PLA and PLA/TPU systems. The addition of $1 \%$ HNT increases toughness, which indicates synergy between the components leading to enhanced energy absorption found also in many analogous NCs by other authors. The subsequent decrease is also relatively low. As a result, a system with favourably balanced mechanical properties has been obtained. Surprisingly, the toughness of the oMMT-containing system is reduced significantly in contrast to the TPU-free NC with a slight increase of toughness. The main reason seems to be a relatively high stiffening of TPU by the significant amount of oMMT localized in this phase. The results indicate that the effect of nanofillers on multiphase systems is quite complex with even antagonistic effects possible.

\section{Acknowledgements}

This work was supported by the Czech Science Foundation (Grant No. 13-15255S).

\section{Notes and references}

1 K. Hamad, M. Kaseem, H. W. Yang, F. Deri and Y. G. Ko, eXPRESS Polym. Lett., 2015, 9, 435.

2 K. S. Anderson, K. M. Schreck and M. A. Hillmyer, Polym. Rev., 2008, 48, 85.

3 Y. Li and H. Shimizu, Macromol. Biosci., 2007, 7, 921.

4 V. Jašo, M. Cvetinov, S. Rakić and Z. S. Petrović, J. Appl. Polym. Sci., 2014, 131, 41104.

5 S. M. Lai, Y. C. Lan, W. L. Wu and Y. J. Wang, J. Appl. Polym. Sci., 2015, 132, 42322.

6 F. Zhao, H. X. Huang and S. D. Zhang, J. Appl. Polym. Sci., 2015, 132, 42511.

7 J. B. Zeng, K. A. Li and A. K. Du, RSC Adv., 2015, 5, 32546.

8 H. Balakrishnan, I. Masoumi, A. A. Yussuf, M. Imran, A. Hassan and M. U. Wahit, Polym.-Plast. Technol. Eng., 2012, 51, 19.

9 M. Y. Gelfer, H. H. Song, L. Liu, B. S. Hsiao, B. Chu, M. Rafailovich, M. Si and V. Zaitsev, J. Polym. Sci., Part B: Polym. Phys., 2003, 41, 44.

10 J. Rotrekl, L. Matějka, L. Kaprálková, A. Zhigunov and J. Hromádková, eXPRESS Polym. Lett., 2012, 6, 975.

11 I. Kelnar, V. Sukhanov, J. Rotrekl and L. Kaprálková, J. Appl. Polym. Sci., 2010, 116, 3621.

12 I. Kelnar, J. Rotrekl, L. Kaprálková, J. Hromádková and A. Strachota, J. Appl. Polym. Sci., 2012, 125, 3477.

13 R. N. Darie, E. Pâslaru, A. Sdrobis, G. M. Pricope, G. E. Hitruc, A. Poiată, A. Baklavaridis and C. Vasile, Ind. Eng. Chem. Res., 2014, 53, 7877.

14 I. Kelnar, V. Khunová, J. Kotek and L. Kaprálková, Polymer, 2007, 48, 5332.
15 J. Odent, Y. Habibi, J. M. Raquez and P. Dubois, Compos. Sci. Technol., 2013, 84, 86.

16 Y. Y. Shi, W. B. Zhang, J. H. Yang, T. Huang, N. Zhang, Y. Wang, G. P. Yuan and C. L. Zhang, RSC Adv., 2013, 3, 26271.

17 H. Xiu, H. W. Bai, C. M. Huang, C. L. Xu, X. Y. Li and Q. Fu, eXPRESS Polym. Lett., 2013, 7, 261.

18 H. Xiu, C. Huang, H. Bai, J. Jiang, F. Chen, H. Deng, K. Wang, Q. Zhang and Q. Fu, Polymer, 2014, 55, 1593.

19 V. Khunova, J. Kristof, I. Kelnar and J. Dybal, eXPRESS Polym. Lett., 2013, 7, 471.

20 V. Khunova, I. Kelnar, J. Kristof, J. Dybal, J. Kratochvíl and L. Kaprálková, J. Therm. Anal. Calorim., 2015, 120, 1283.

21 I. Kaygusuz and C. Kaynak, Plast., Rubber Compos., 2015, 44, 41.

22 M. Liu, Y. Zhang and C. Zhou, Appl. Clay Sci., 2013, 75-76, 52.

23 B. J. Rashmi, K. Prashantha, M. F. Lacrampe and P. Krawczak, eXPRESS Polym. Lett., 2015, 9, 721.

24 W. L. Tham, Z. A. Mohd Ishak and W. S. Chow, J. Macromol. Sci., Part B: Phys., 2014, 53, 371.

25 P. Pal, M. Kundu, A. Malas and C. Das, J. Appl. Polym. Sci., 2014, 131, 39587.

26 M. Poikelispää, A. Das, W. Dierkes and J. Vuorinen, J. Appl. Polym. Sci., 2013, 127, 4688.

27 P. Pal, M. K. Kundu, S. Kalra and C. K. Das, Open J. Appl. Sci., 2012, 2, 277.

28 W. L. Tham, B. T. Poh, Z. A. Mohd Ishak and W. S. Chow, J. Appl. Polym. Sci., 2015, 133, 42850.

29 L. T. Lim, R. Auras and M. Rubino, Prog. Polym. Sci., 2008, 33, 820.

30 J. Moll and S. K. Kumar, Macromolecules, 2012, 45, 11311135.

31 S. M. Lai, S. H. Wu, G. G. Lin and T. M. Don, Eur. Polym. J., 2014, 52, 193.

32 E. H. Kerner, Proc. Phys. Soc., 1956, 69, 808.

33 S. N. Bhattacharya, R. K. Gupta and M. R. Kamal, Polymeric Nanocomposites, Hanser, Munich, 2008.

34 H. Balakrishnan, A. Hassan, M. U. Wahit, A. A. Yussuf and S. B. Abdul Razak, Mater. Des., 2010, 31, 3289.

35 H. Balakrishnan, I. Masoumi, A. A. Yussuf, M. Imran, A. Hassan and M. U. Wahit, Polym.-Plast. Technol. Eng., 2012, 51, 19.

36 H. Bai, C. Huang, H. Xiu, Y. Gao and Q. Zhang, Polymer, 2013, 54, 5257.

37 M. A. Hood, B. B. Wang, J. M. Sands, J. J. La Scala, F. L. Beyer and C. Y. Li, Polymer, 2010, 51, 2191.

38 V. Pistor, D. de Conto, F. G. Ornaghi and A. J. Zattera, J. Nanomater., 2012, 2012, 283031.

39 P. J. Pan, B. Zhu, W. H. Kai, T. Dong and Y. Inoue, Macromolecules, 2008, 41, 4296. 